\title{
Interactive comment on "Microbial
} \section{diversity-informed modelling of polar marine} ecosystem functions" by Hyewon Heather Kim et

\section{al.}

Hyewon Heather Kim et al.

hkim@whoi.edu

Received and published: 31 March 2021

Thank you very much for your constructive comments and suggestions on our manuscript. Please note four major elements as our effort to fully address your and other reviewer's concerns together, which served as the basis for updated results in the revised manuscript as well as our response to each comment in this file. After these major changes summarized below, most of the results and main conclusions remained similar compared to the previous version: 1) significant associations of the observed bacterial mode with the modelled NPP, POC flux, and BCD; 2) significant associations of the observed fHNA with the modelled NPP, POC flux, and BCD 3) larger 
increases of HNA stocks and functions under climate change conditions than those of LNA cells; and 4) larger cell-specific BP and SDOC uptake rates of HNA cells than those of LNA cells. This suggests the robustness of our model study.

In response to the reviews, we made a number of substantial revisions to the modeling study and manuscript:

1) Modification of modeling framework: We re-built, re-optimized, and re-analyzed the model by completely changing the previous version's 0-D (fixed surface layer) formulation to a 1-D (vertical profile) framework.

2) Additional data assimilation: We added diatom and cryptophyte Chl observations for 2010-2011, 2012-2013, and 2013-2014 in that data assimilation; this new data became available for use during the period of revision.

3) Model equations and GMD manuscript: We included a complete set of model equations (line 92-104, Appendix A) and other details about the model set-up (Text S1-4), as well as attached our Geoscientific Model Development (GMD) manuscript on the original WAP model that served as the basis for our study's bacteria-oriented model (Kim, H. H., Luo, Y.-W., Ducklow, H. W., Schofield, O. M., Steinberg, D. K., and Doney, S. C.: WAP-1D-VAR v1.0: Development and Evaluation of a One-Dimensional Variational Data Assimilation Model for the Marine Ecosystem Along the West Antarctic Peninsula, Geosci. Model Dev. Discuss. [preprint], https://doi.org/10.5194/gmd-2020-375, in review, 2021).

4) Climate change simulations: We updated error estimates in the climate change experiments (Results 3.4) after fixing an error in the Monte Carlo simulation code. Temperature and sea-ice perturbations were also replaced by $+0.5^{\circ} \mathrm{C}$ and $+1.0^{\circ} \mathrm{C}$ of warming and $5 \%$ and $10 \%$ of melting, from $+1.0^{\circ} \mathrm{C}$ and $+2.0^{\circ} \mathrm{C}$ of warming and $10 \%$ and $20 \%$ of melting in the previous version, in order to reflect better the trends and changes relevant to the WAP. 
5) Others: We 1) added the summary of the climatological model optimization in Table 2 (missing in the previous version), 2) combined the 4-modelled years together (Figure 3a; each year presented in the previous version) and included the Taylor diagram of the climatological model (Figure 3b; missing in the previous version) for model skill assessment, 3) removed discussion on microzooplankton model fits from Table 1 for consistency (presented in Table 1 in the previous version but never discussed), and 4) removed the discussion on the fate of $B C D$ as it did not add new information to the study.

In particular, modification of the modeling framework to 1-D vertical profile and additional data assimilation were both labor-intensive and time-consuming, which caused a long delay in providing our Final Author's comments. Thank you again for your patience and for willingness to re-review the revised manuscript in advance. Below are our responses to each of your specific comments that are highlighted throughout the revised manuscript file.

This ms presents a bacteria-oriented ecosystem model, calibrated with a data assimilation scheme, with two explicit bacteria types, fast growing (HNA) and slow growing (LNA). The authors find that properties of the bacterial community are strong predictors of bacterial carbon (C) demand, primary production (PP), and export (EP). The calibrated model is used to make predictions for a warming ocean. At first I was quite intrigued by the approach of this study. But after going through the ms, it now appears fraught with too many problems to make it worthwhile. The problems start already with the title. I consider "microbial diversity-informed modelling..." a gross overstatement of the authors' approach, which is more correctly describes in the abstract as "bacteriaoriented". Below I will outline why I consider this a failed attempt and how it might be modified into a useful contribution. Because I have the strong impression that essential information about the model and the data-assimilation method is missing, I will not go into much detail, though.

Answer: We hope that the GMD manuscript and added details about the model in the 
revised version clear up these concerns. We agree with your assessment on the title of the study. With only part of the microbial diversity data directly informing the model (bacterial physiology data) we modified the title to "Modelling polar marine ecosystem functions guided by bacterial physiological and taxonomic traits" in the revised version.

My first major problem was understanding the design of the model. The authors refer to one published work (Luo et al., 2010) regarding the model equations (besides unpublished manuscripts, which may or may not eventually be published), and present only the equations for the two bacteria groups. The model of Luo et al. (2010) is much more complex, totalling 30 state variables, than this one (with 12 states), so this reference does not really help much. Without access to the model equations, any attempt to understand the model code will be futile. In consequence, it also remains unclear what the model currency is. According to Fig. 1 and the description in the text (which is not very clear in this respect, except that the number of states is 12) the model employs a fixed stoichiometry approach but it remains unclear whether the fluxes are based on nitrogen $(\mathrm{N})$ or phosphorus $(\mathrm{P})$. Also according to Fig. 1, it appears that inorganic nutrient have no effect on and are not utilised by phytoplankton, leaving open the question what drives PP in this model. Since only very little information about the model is provided in the text of the ms and the supplement, the model design remains very much opaque. From what little information is presented I can see clearly only that the model is $0-\mathrm{D}$ and employs a rather simplistic physiology (fixed stoichiometry).

Answer: It is correct that the model was applied as a 0-D framework in the previous version (i.e., a 0-D box model of the surface layer at $10 \mathrm{~m}$ ), but as mentioned above, the revised version is now based on the new model results from a 1-D vertical profile framework (line 126-129). The model tracks and simulates C, N, and P stocks as concentrations in different inorganic and organic pools (e.g., C, N, P for all living model groups, N for nitrate and ammonium, and $\mathrm{P}$ for phosphate). The model has flexible stoichiometry, in which phytoplankton store more $\mathrm{C}$ under high light and nutrient-depleted conditions and more $\mathrm{N}$ and $\mathrm{P}$ under low light conditions. If the $\mathrm{N}$ or $\mathrm{P}$ cellular quota is lower 
than the predefined reference (Redfield) ratio, plankton excrete DOM to adjust their stoichiometry close to the reference ratio. To make these points clear, we 1) revised Figure 1 to show $\mathrm{N}$ and $\mathrm{P}$ uptake by phytoplankton and 2) added a section demonstrating the model's variable stoichiometry (Text S1, line 224-230). The model PP is driven by photosynthetic active radiation and nutrient uptake by phytoplankton, but given the abundance of NO3, PO4, and $\mathrm{SiO} 3$ (Kim et al., 2016, doi: 10.1002/2015JG003311) and iron (Annett et al., 2017, doi: 10.1016/j.marchem.2017.06.004) at the study site, it is the light level that primarily limits PP. To make this pint clear, we added the model coupled ordinary differential equations (line 92-103, Appendix A) and other details (Text S1-4).

The model has 84 parameters, of which 22 (inferred from I. 219 of the ms) are calibrated via data assimilation. What is missing here is a description of how these 22 parameters have been selected in the first place. For example, was the selection based on a preliminary sensitivity analysis or a-priori knowledge or assumptions of the model equations? Also, 22 is, in my experience, a very large number of parameters to constrain given the kinds and amount of data employed here. Thus, it is not very surprising that only a subset of 7-10 of these could be constrained well.

Answer: We revised the Material and Methods 2.3 to demonstrate how we chose an initial subset of model parameters submitted to optimization (line 156-165). With regard to your concern on too many optimized parameters, the total number of optimized parameters changed from 12-15 in the previous version to 3-6 in the revised version, while the total number of constrained (optimized with low uncertainties) parameters changed from 7-10 in the previous version to 5-7 in the revised version, therefore, the revised version has a larger fraction of well-constrained parameters.

Related to this, the next problem is the description regarding overfitting and portability. I agree that these are essential concepts all too often neglected in modelling studies and so was happy to see that these are addressed here. Nevertheless, I question the quantification of overfitting (lines 175-179) by comparing the residual error with the 
(undefined in the ms) "target error" of the observations. Overfitting has very little to do with the noisiness of the observations. It is a consequence of the fact that every model is a simplification of the system it describes, and it is also tightly related to portability. The connection is that overfitting can compromise portability, and this is a good way of assessing overfitting. Overfitting often results from attempting to constrain too many parameters, which is revealed here by several parameters being not well constrained (Tables S2-S6). The different estimates of portability for the different year are another indication of overfitting.

Answer: This is an excellent point. The portability analysis showed that the optimized model parameter set for 2012-13 was the most portable while the parameter set for 2011-12 was least portable (Table 2), in which the most ( $n=7$ out of total 11 ) and the least numbers ( $n=5$ out of total 11 ) of parameters were constrained (i.e., optimized with low uncertainties), respectively (Tables S3-4). The other two years exhibited intermediate levels of portability, with similar portability index values characterized by the same number of constrained parameters ( $n=6$ out of total 10 for 2010-11 and $\mathrm{n}=6$ out of total 12 for 2013-14; Tables S2, S5). In other words, it was the number of well-constrained parameters that mattered most in driving high model portability, suggesting the connection between overfitting and portability of optimized models, as you suggested. It is commonly thought that more tuned parameters indicate a higher possibility of overfitting and less portability. However, our analysis showed that if the tuned parameters were well-constrained by observations, they would not compromise portability. We added these points in Discussion 4.1 (line 370-376). However, it is our understanding that a reduced chi-square estimate of model fit is a reasonable metric for assessing overfitting of data (Glover et al., 2011, doi: 10.1017/CBO9780511975721). Target errors in our study reflect both the observational errors and seasonal and interannual variations of the observations (which has been intensively defined and discussed in Section 2.5), and larger target errors compared to model-observation misfits, or the noisiness of the observations, would be an indication of overfitting. 
I must admit that the concept of the bacterial modes was new to me, so I was happy to see the clear definition in Section 2.2 (first para). However, I could not figure out the main characteristic of these modes, since only very cursory information is presented in the text and Fig. 6. A table listing the modes and their properties and composition might be very helpful here. As it stands, the concept remains rather confusing. For example, the authors state that (I. 276) each mode is dominated by unique bacterial taxa. But considering Fig. 6, it appears that Candidatus Pelagibacter dominates both modes 6 and 1 , although it appears that mode 1 is supposed to be dominated by Candidatus Thioglobus.

Answer: Thank you for this comment. The modes are entirely taxonomic, although they can be related statistically to different physiological or ecophysiological parameters. A complete discussion of the modes is given in Bowman et al. (2017; Bowman, J., Amaral-Zettler, L., J Rich, J. et al. Bacterial community segmentation facilitates the prediction of ecosystem function along the coast of the western Antarctic Peninsula. ISME J 11, 1460-1471, doi: 10.1038/ismej.2016.204). Because of this we have opted not to give a more in-depth description here, but have modified the caption for Fig. 6 to try and make this concept more clear. In direct response to your question Mode 6 is dominated by P. ubique (comprising over $50 \%$ of the community for some map units in Mode 6) while Mode 1 is dominated by T. singularis (also reaching over $50 \%$ of the community).

The above may be viewed as more technical problems, which could possibly be dealt with by, e.g., a detailed model description with all equations, or a recalibration of the model, etc. However, I also see a major conceptual problem regarding the design of the study. The problem lies in the way the authors use the model to make predictions for a warmer ocean. The main assumption behind the presented approach is that bacterial community composition is strongly correlated ("strong predictor", Abstract) to PP and EP. The functional bacterial community composition is represented in the model and its calibration by assigning higher growth rates to HNA than LNA. Nevertheless, bacteria 
process the DOM produced during PP, so the behaviour of the bacterial community must be viewed as a response, not a driver, of PP. If bacterial community composition is in fact strongly correlated with PP and EP, that is in itself a very significant finding and I would very much like to see this substantiated. It could become a very useful diagnostic tool. However, here the authors treat the bacteria as the driving force determining PP and EP, which is wrong for several reasons. First, it reverses the cause-effect relation between bacterial activity and PP. Second, even if the cause-effect relation was OK, the data do not cover sufficient interannual temperature variability to allow predicting the response to a warmer ocean.

Answer: Thank you for this insightful comment. It is well understood that there can be significant feedback between bacteria and PP, in particular in systems where large PP rates are supported by fast/efficient recycling of nutrients by bacterial remineralization of organic matter to inorganic nutrients. By contrast, the fact that macro- and micronutrients are abundant at the study site (Kim et al., 2016, doi: 10.1002/2015JG003311; Annett et al., 2017, doi: 10.1016/j.marchem.2017.06.004) makes the limitation of light, not of nutrients (Eq. A.2.4-5, B.2.4-5) determine PP rates (Eq. A.2.7, B.2.7). PP rates are also proportional to phytoplankton biomass that is grazed by microzooplankton which follows preferential selection on phytoplankton (i.e., gDA, gCR) versus bacterial food sources (i.e., gHNA, gLNA) as well as bacterial biomass (Eq. A.2.33, A.3.33). This step-wise, indirect connection makes the bacterial-microzooplankton grazing influence on PP rather remote compared to nutrient recycling by bacteria. The same applies to EP. Thus, we agree with your argument that bacteria should be regarded as a responder, rather than a driver, of $\mathrm{PP}$ and EP, and therefore changed the wording from "predict/predictor" to "indicate/indicator", "respond", "reflect", or "associated" in the revised version. Regarding your second comment, our climate change experiments identified functional relationships between modeled processes and temperature. Although the real response may not follow the same functions when temperature increases beyond the existing observed range, this is the best we can do for future projections as in other modeling studies. In our experiments, 
the "climate change" condition is equivalent to simultaneous warming and melting, which have a more solid physical basis as well as more profound impacts on bacterial processes than the warming alone condition does. Nevertheless, we reduced the range of warming to $+0.5^{\circ} \mathrm{C}$ and $+1.0^{\circ} \mathrm{C}$, which are relatively minor increases that are not expected to cause very different trends from the existing observed patterns.

Please also note the supplement to this comment:

https://bg.copernicus.org/preprints/bg-2020-302/bg-2020-302-AC1-supplement.pdf

Interactive comment on Biogeosciences Discuss., https://doi.org/10.5194/bg-2020-302, 2020. 